\title{
Design of 2:4 and 3:8 decoder circuit using QCA technology
}

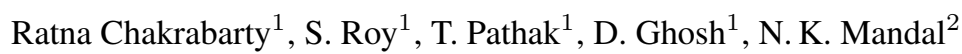 \\ ${ }^{1}$ Institute of Engineering \& Management, Department of Electronics \& Communication Engineering, \\ Salt Lake Electronics Complex, Sector V, Kolkata, 700091, India \\ ${ }^{2}$ University of Engineering \& Management, Department of Electronics \& Communication Engineering, \\ Salt Lake Electronics Complex, Sector V, Kolkata, 700091, India \\ ratna.chakrabarty@iemcal.com,soumyaruproy98@gmail.com,trambak.jaku@gmail.com, \\ debanjanghosh.bwn@gmail.com, niranjanmandal54@gmail.com
}

DOI 10.17586/2220-8054-2021-12-4-442-452

Quantum Dot Cellular Automata is an emerging technology in the field of nanotechnology and has the potential to replace the existing CMOS technology. CMOS technology has its limitations in terms of high leakage current. However, QCA technology has higher speed of operation and very low power consumption. In this paper, designs for 2-4 and 3-8 decoder circuits have been made using a novel inverter circuit design which helps in decreasing the energy dissipation of the circuits. Finally, the proposed designs are compared to previously made designs. All the circuits in this paper have been simulated using QCA Designer software.

Keywords: QCA, decoders, laser ablation, majority voter, kink energy.

Received: 25 May 2021

\section{Introduction}

The QCA technology is a transistorless technology which is superior to the existing CMOS technology in terms of power consumption, area coverage and speed of operation. Many basic electronic components which are essential have been made using the QCA technology, namely adders [1-3], subtractors [1,3], multiplexers [4-7], decoders [8,9] and memory elements $[10,11]$. This technology uses the Coulombic forces between charged particles. Unlike the CMOS technology, in QCA, they transfer the polarization of each cell [12]. Hence, polarization of QCA cell plays a very important role in choosing the perfect QCA device for use. There are two polarizations, $P=+1$ and -1 as shown in Fig. 1 which are treated as logic 1 and logic 0 respectively.

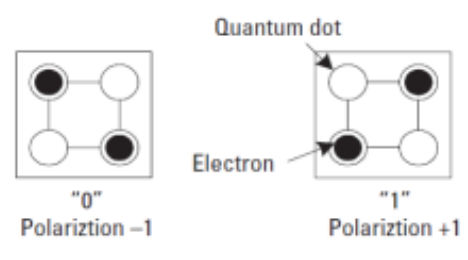

FIG. 1. QCA cell with the polarizations

The basic gate in this technology is a majority voter gate [13], as shown in Fig. 2, which can be programmed to act as an AND gate or an OR gate, depending on the control value given. Any one of the inputs can be used as a control input and the remaining two can act as normal inputs. When the control input is given as +1 the majority voter acts as a OR Gate and when the control input is given as -1, the majority voter acts as an AND Gate.
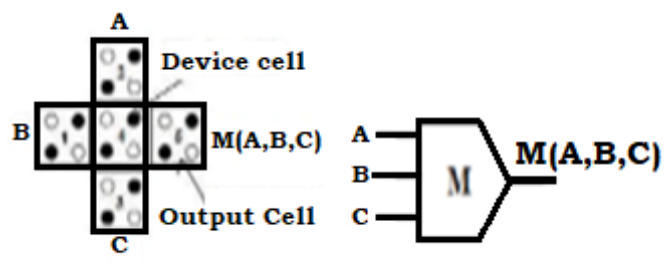

FIG. 2. Majority voter gate 
One important aspect in this QCA technology is the concept of clocks shown in Fig. 3. They are used to synchronize the whole circuit and provide the direction of state transition. There are four clocks, namely clock 0 , clock 1, clock 2 and clock 3 and each of them has four clocking zones, switch, hold, release and relax. During the switch phase, the inter-dot potential barrier becomes high and the cell becomes polarized based on the polarization state of its driver cell. In the hold phase, the barrier remains high as the cells preserve their state. In the release phase, the cells become unpolarized as the potential barrier decreases and in relax phase they remain unpolarized [12,14].

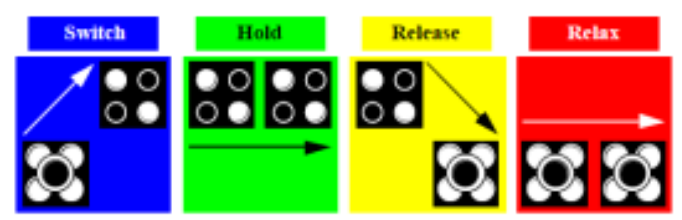

FIG. 3. The clocking zones

In the subsequent sections of the paper, we have designed a 2:4 decoder and a 3:8 decoder with an efficient architecture using QCA technology. The designs are made such that it can be scaled up from 2:4 to 3:8 to 4:16 decoders and so on. Finally, we compare it to the previous designs as mentioned in [15-18].

\section{2:4 Decoder circuit}

Figure 4 is the proposed design of 2:4 decoder circuit using QCA Designer tool [19]. The whole circuit is designed in a single layer only. The design consists of 50 cells and occupies the area of $0.016 \mu \mathrm{m}^{2}$. The output of this decoder is shown in Fig. 5 and the truth table is shown in Table 1.

TABLE 1. Truth table for 2:4 decoder

\begin{tabular}{|cc|cccc|}
\hline \multicolumn{2}{|c|}{ INPUT } & \multicolumn{4}{c|}{ OUTPUT } \\
\hline$S_{1}$ & $S_{0}$ & $I_{0}$ & $I_{1}$ & $I_{2}$ & $I_{3}$ \\
\hline \hline 0 & 0 & 1 & 0 & 0 & 0 \\
\hline 0 & 1 & 0 & 1 & 0 & 0 \\
\hline 1 & 0 & 0 & 0 & 1 & 0 \\
\hline 1 & 1 & 0 & 0 & 0 & 1 \\
\hline
\end{tabular}

It is evident from Fig. 6(a), that the polarization decreases as the temperature increases. For the output to be within tolerable ranges, the temperature of operation of the circuit is 1 to $9 \mathrm{~K}$. Within that temperature frame, the lowest energy recorded is $0.0237 \mathrm{eV}$ as shown in Fig. 6(b).

Calculation of kink energy is important in QCA-based design circuit. Kink energy is the energy difference between two neighboring or adjacent cells. Kink energy between two cells depends on the dimension of the QCA cell as well as the spacing between adjacent cells. It is independent of temperature. It is one of the most significant parameters for the stability of the design. The state having minimum Kink Energy is most stable state.

The kink energy (in Joule) between two electron charges is calculated using the formula:

$$
U=\frac{k Q_{1} Q_{2}}{2}
$$

where $k=1 /\left(4 \pi \epsilon_{0} \epsilon_{r}\right)=9 \times 10^{-9}, Q_{1}=Q_{2}=1.6 \times 10^{-19} \mathrm{C}-$ charge of an electron.

$$
U=\frac{23.04 \times 10^{-29}}{r}
$$

$r$ - distance between the two charges.

$$
U_{T}=\sum_{i} U_{i}
$$

$U_{T}$ - summation of all the individual kink energies (in Joule).

For this calculation, the below postulates are considered. 


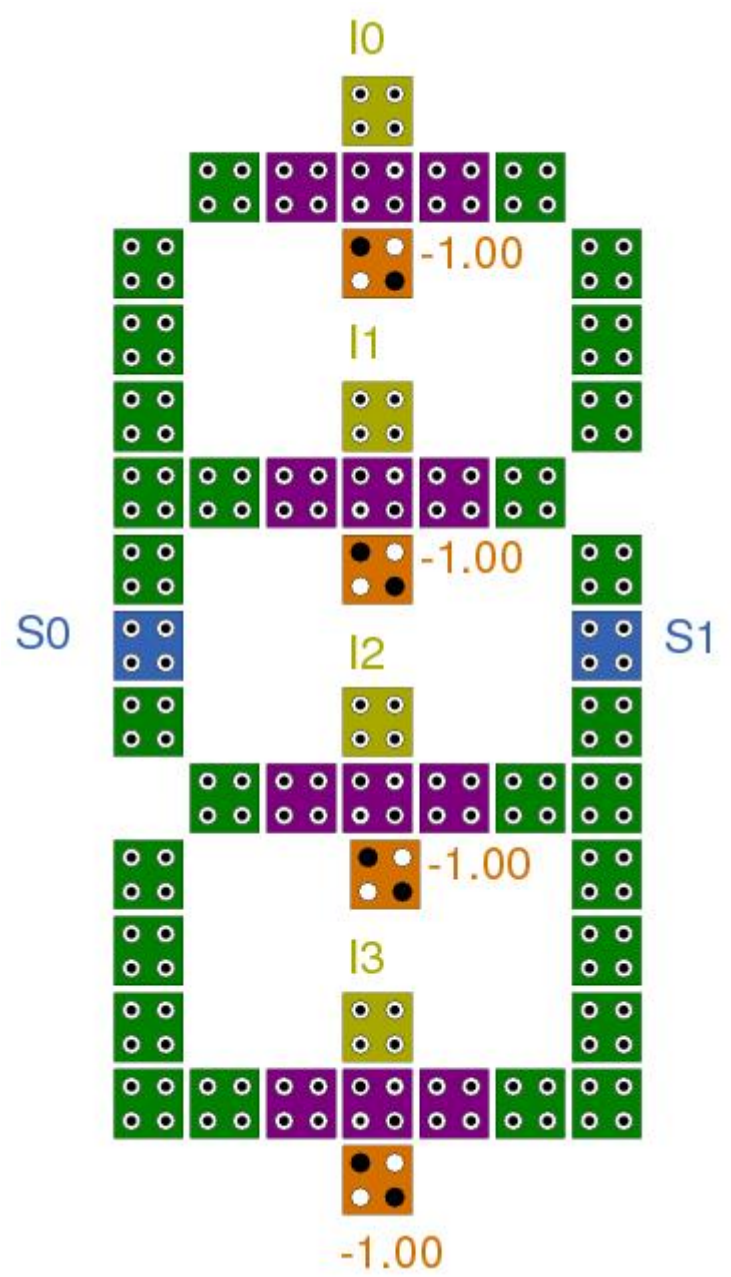

FIG. 4. Proposed 2:4 decoder

(1) All cells are alike and the distance of end to end of each cell is $18 \mathrm{~nm}$ as shown in Fig. 7.

(2) The space between two neighbouring cells (interspacing distance) is $2 \mathrm{~nm}$.

(3) The diameter of each quantum dot is $2.5 \mathrm{~nm}$.

(4) The distance between the two layers used for the design is $11.5 \mathrm{~nm}$.

Below, a particular value of the two inputs is taken and the kink energy of all the corresponding output cells are calculated. Here, $S_{1}=1$ and $S_{0}=0$ is considered for the kink energy calculation. As per the truth table of 2:4 Decoder, for input $\left(S_{1} S_{0}\right)=(10)$, the outputs are $I_{0}=0, I_{1}=0, I_{2}=1$ and $I_{3}=0$. The yellow cells are the output cells. As it can be observed from Fig. 8, the $I_{2}$ is only high and the rest are low. All the electrons (black dots) are arranged such that minimal possible energy configuration is achieved. Then the kink energies are calculated. Table 2 shows the kink energies for the proposed 2:4 decoder circuit.

\section{3:8 Decoder circuit}

Figure 9 shows the 3 layered 3:8 decoder circuit. The 3:8 decoder can be derived from the 2:4 circuit. The three layers of the decoder circuit is shown in Fig. 10.

Figure 11 shows the simulated output for the 3:8 decoder circuit and Fig. 12 shows the polarization and energy dissipation graph with the temperature variation. Below, a particular value of the three inputs is taken and the kink energy of all the corresponding output cells are calculated. Here, $S_{2}=1, S_{1}=1$ and $S_{0}=0$ is considered for the kink energy calculation. As per the truth table of Table 3 of 3:8 Decoder, let the input $\left(S_{2} S_{1} S_{0}\right)=(110)$, the outputs are $I_{0}=0, I_{1}=0, I_{2}=0, I_{3}=0, I_{4}=0, I_{5}=0, I_{6}=1$ and $I_{7}=0$. The yellow cells are the output cells. As it can be observed from Fig. 13, the $I_{6}$ is only high and the rest are low. Again, all the electrons (black dots) are arranged such that minimal possible energy configuration is achieved just like the 2:4 decoder case. Then the kink energies are 


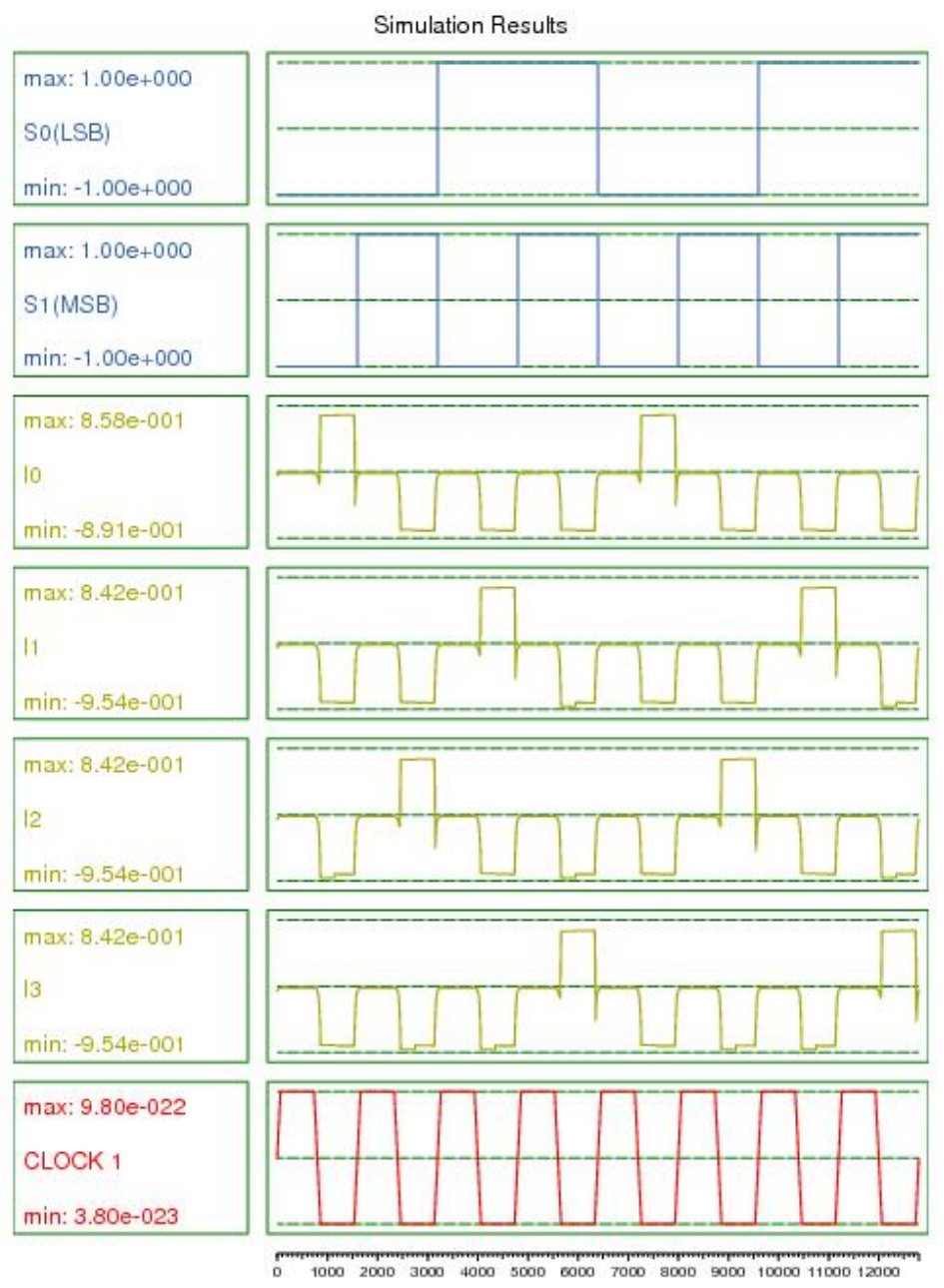

FIG. 5. Output of proposed 2:4 decoder circuit
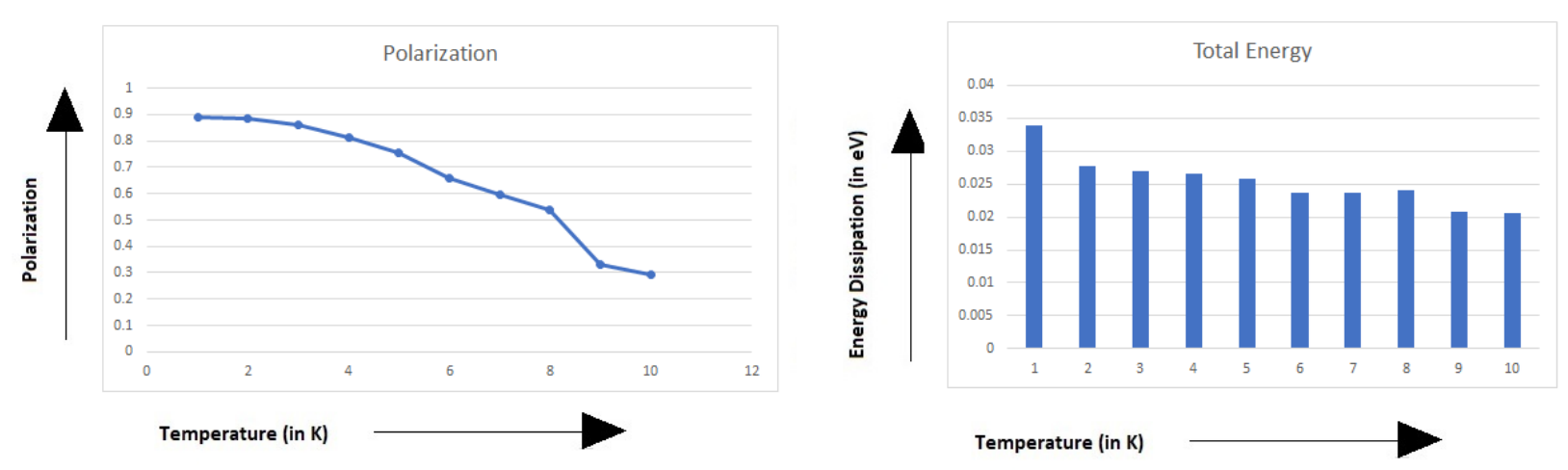

FIG. 6. Polarization (a) or energy dissipation (b) vs temperature 


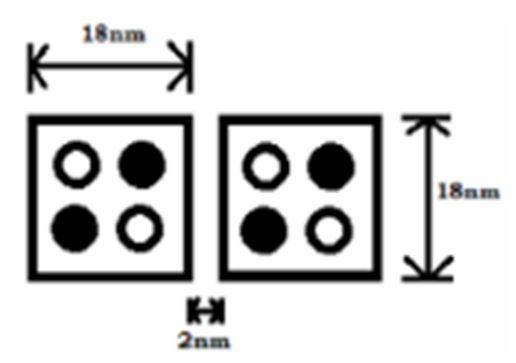

FIG. 7. Dimensions of the QCA cells

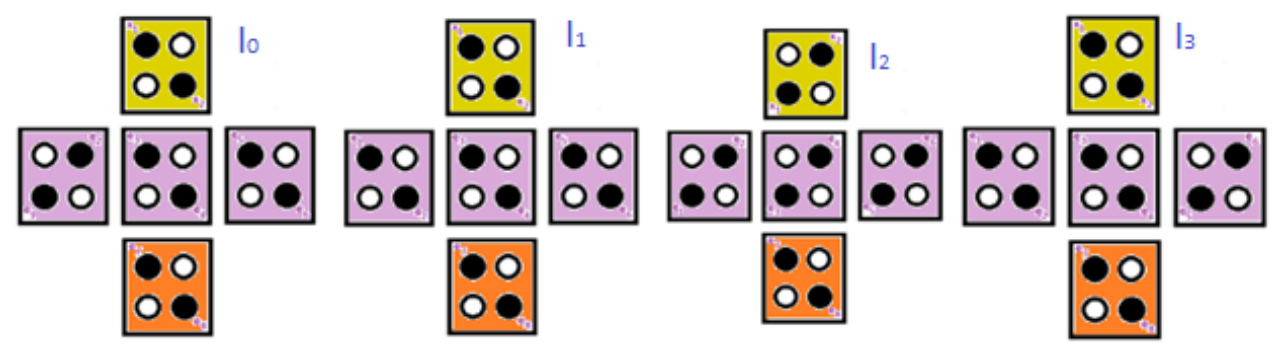

FIG. 8. Kink energy calculation diagram reference (from left to right $I_{0}, I_{1}, I_{2}, I_{3}$ )

TABLE 2. Kink Energy for 2:4 decoder circuit

\begin{tabular}{|c|cc|cc|cc|cc|}
\hline & \multicolumn{2}{|c|}{$I_{0}\left(\times 10^{-21}\right)$} & \multicolumn{2}{|c|}{$I_{1}\left(\times 10^{-21}\right)$} & \multicolumn{2}{|c|}{$I_{2}\left(\times 10^{-21}\right)$} & \multicolumn{2}{|c|}{$I_{3}\left(\times 10^{-21}\right)$} \\
\cline { 2 - 10 } & $\mathrm{x} 1$ & $\mathrm{x} 2$ & $\mathrm{x} 1$ & $\mathrm{x} 2$ & $\mathrm{x} 1$ & $\mathrm{x} 2$ & $\mathrm{x} 1$ & $\mathrm{x} 2$ \\
\hline \hline$U_{e 1}$ & 6.54 & 6.54 & 8.15 & 7.42 & 8.15 & 5.62 & 8.15 & 7.42 \\
\hline$U_{e 2}$ & 10.08 & 10.08 & 7.42 & 8.15 & 14.81 & 8.15 & 7.42 & 8.15 \\
\hline$U_{e 3}$ & 11.52 & 16.21 & 11.52 & 16.21 & 11.52 & 7.59 & 11.52 & 16.21 \\
\hline$U_{e 4}$ & 7.59 & 11.52 & 7.59 & 11.52 & 16.21 & 11.52 & 7.59 & 11.52 \\
\hline$U_{e 5}$ & 8.15 & 14.81 & 8.15 & 14.81 & 8.15 & 7.42 & 8.15 & 10.08 \\
\hline$U_{e 6}$ & 5.62 & 8.15 & 5.62 & 8.15 & 7.42 & 8.15 & 6.54 & 10.08 \\
\hline$U_{e 7}$ & 5.76 & 7.14 & 5.76 & 7.14 & 7.43 & 5.62 & 5.76 & 7.14 \\
\hline$U_{e 8}$ & 4.62 & 5.76 & 4.62 & 5.76 & 5.62 & 4.70 & 4.62 & 5.76 \\
\hline Total $\left(U_{T}\right)$ & 59.88 & 80.21 & 58.83 & 79.16 & 79.31 & 58.77 & 59.75 & 76.36 \\
\hline
\end{tabular}

calculated. Table 4 and 5 shows the kink energies for the proposed 3:8 decoder circuit. Now, $I_{1}, I_{2}$ and $I_{3}$ has 16 possible interactions with the electrons, so there are 16 rows in their case (Table 5), and the remaining have 8 rows (Table 4). From Fig. 13, it is also clear that due to the interaction of the output cell electrons with the main cell layer electrons, the calculations of kink energies for $I_{1}, I_{2}$ and $I_{3}$ are a bit complex.

\section{Observation and results}

Table 6 summarizes few of the parameters of the existing 2:4 decoders. It is a comparison between the already available decoder circuits and our proposed 2:4 decoder circuit design. In terms of cell count, layer separation and consumption of area proposed 2:4 decoder is better design compare to the 3 layered circuits proposed in [15] and [16]. Though the papers in [17] and [18] had designed a single layered circuit but number of cells and area requirement is more than the proposed design. Due to this single layer, the space occupied by our proposed circuit is much less. Also, this structure can be further extended to make a 3:8 decoder circuit. And, because of this single layered circuit, the 


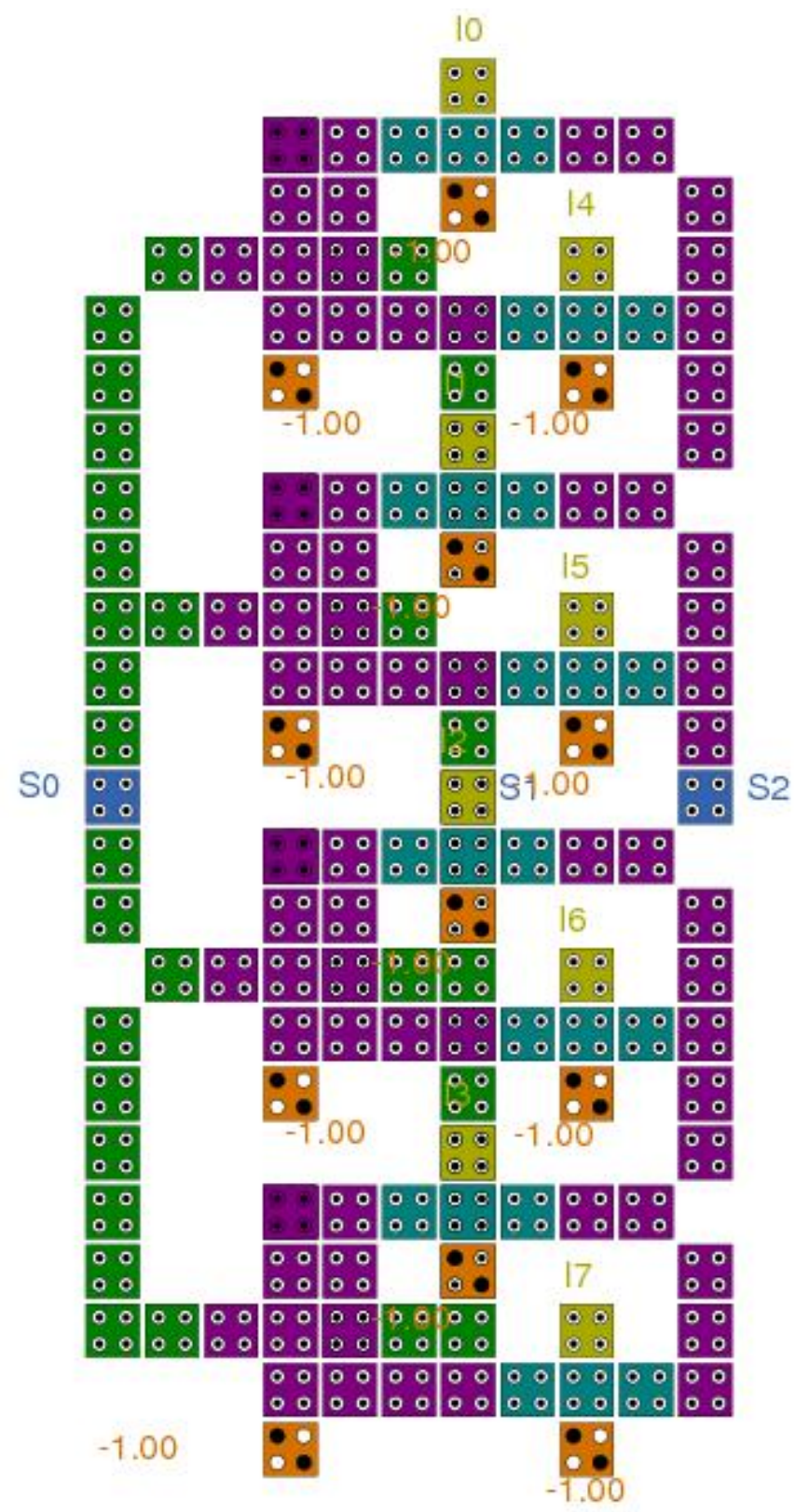

FIG. 9. Proposed 3:8 decoder (3 layered circuit)

cost of fabricating the circuit is much less. Table 7 shows the comparisons among the 3:8 decoders with the proposed decoder.

\section{Conclusion}

There are few decoder circuits designed in QCA. The work in [15] is slightly efficient design in terms of the cells count for the 3:8 decoder circuit but the layers used for the design and the consumption of area is same with the proposed design. The proposed design can be formed from 2:4 decoder and can be easily scaled up to form 3:8, 4:16 decoders and so on. Designing higher complex decoders will be easier with the proposed design. 


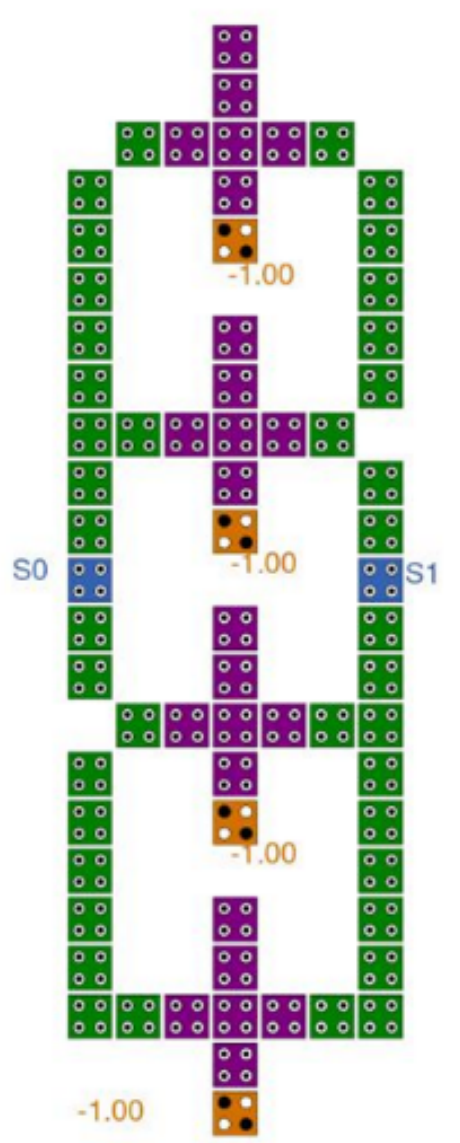

Main Cell Layer

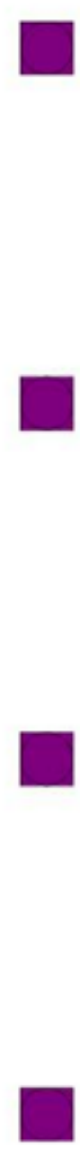

Layer 1(Via Layer)

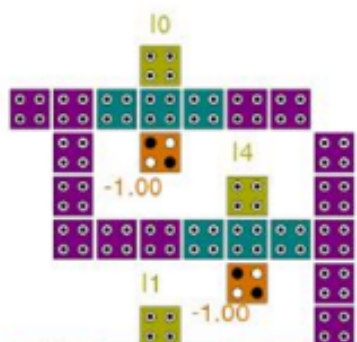

$\because \because: \%: \because: 0 \%$
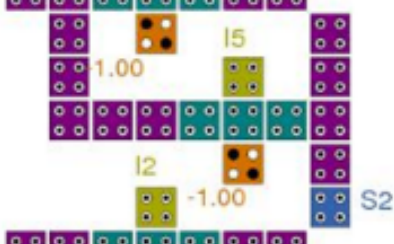

$\therefore$
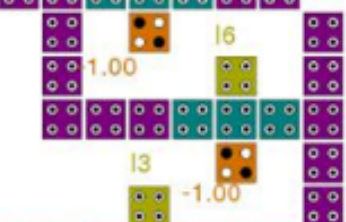

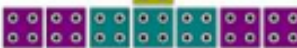

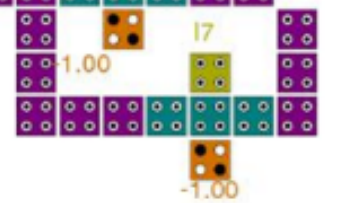

Layer 2

FIG. 10. Breakup of the 3 layers in the circuit

TABLE 3. Truth table for 3:8 decoder

\begin{tabular}{|ccc|cccccccc|}
\hline \multicolumn{7}{|c|}{ INPUT } & \multicolumn{7}{|c|}{ OUTPUT } \\
\hline$S_{2}$ & $S_{1}$ & $S_{0}$ & $I_{0}$ & $I_{1}$ & $I_{2}$ & $I_{3}$ & $I_{4}$ & $I_{5}$ & $I_{6}$ & $I_{7}$ \\
\hline \hline 0 & 0 & 0 & 1 & 0 & 0 & 0 & 0 & 0 & 0 & 0 \\
\hline 0 & 0 & 1 & 0 & 1 & 0 & 0 & 0 & 0 & 0 & 0 \\
\hline 0 & 1 & 0 & 0 & 0 & 1 & 0 & 0 & 0 & 0 & 0 \\
\hline 0 & 1 & 1 & 0 & 0 & 0 & 1 & 0 & 0 & 0 & 0 \\
\hline 1 & 0 & 0 & 0 & 0 & 0 & 0 & 1 & 0 & 0 & 0 \\
\hline 1 & 0 & 1 & 0 & 0 & 0 & 0 & 0 & 1 & 0 & 0 \\
\hline 1 & 1 & 0 & 0 & 0 & 0 & 0 & 0 & 0 & 1 & 0 \\
\hline 1 & 1 & 1 & 0 & 0 & 0 & 0 & 0 & 0 & 0 & 1 \\
\hline
\end{tabular}


TABLE 4. Kink energy for $I_{0}, I_{4}, I_{5}, I_{6}, I_{7}$

\begin{tabular}{|c|cc|cc|cc|cc|cc|}
\hline & \multicolumn{2}{|c|}{$I_{0}\left(\times 10^{-21}\right)$} & \multicolumn{2}{|c|}{$I_{4}\left(\times 10^{-21}\right)$} & \multicolumn{2}{|c|}{$I_{5}\left(\times 10^{-21}\right)$} & $I_{6}\left(\times 10^{-21}\right)$ & $I_{7}\left(\times 10^{-21}\right)$ \\
\cline { 2 - 12 } & $\mathrm{x} 1$ & $\mathrm{x} 2$ & $\mathrm{x} 1$ & $\mathrm{x} 2$ & $\mathrm{x} 1$ & $\mathrm{x} 2$ & $\mathrm{x} 1$ & $\mathrm{x} 2$ & $\mathrm{x} 1$ & $\mathrm{x} 2$ \\
\hline \hline$U_{e 1}$ & 8.15 & 7.42 & 8.15 & 7.42 & 8.15 & 7.42 & 8.15 & 5.62 & 8.15 & 7.42 \\
\hline$U_{e 2}$ & 7.42 & 8.15 & 7.42 & 8.15 & 7.42 & 8.15 & 14.81 & 8.15 & 7.42 & 8.15 \\
\hline$U_{e 3}$ & 11.52 & 16.21 & 11.52 & 16.21 & 11.52 & 16.21 & 11.52 & 7.59 & 11.52 & 16.21 \\
\hline$U_{e 4}$ & 7.59 & 11.52 & 7.59 & 11.52 & 7.59 & 11.52 & 16.21 & 11.52 & 7.59 & 11.52 \\
\hline$U_{e 5}$ & 8.15 & 14.81 & 8.15 & 10.08 & 8.15 & 10.08 & 8.15 & 7.42 & 8.15 & 10.08 \\
\hline$U_{e 6}$ & 7.59 & 8.15 & 6.54 & 10.08 & 6.54 & 10.08 & 7.42 & 8.15 & 6.54 & 10.08 \\
\hline$U_{e 7}$ & 5.76 & 7.14 & 5.76 & 7.14 & 5.76 & 7.14 & 7.43 & 5.62 & 5.76 & 7.14 \\
\hline$U_{e 8}$ & 4.62 & 5.76 & 4.62 & 5.76 & 4.62 & 5.76 & 5.62 & 4.7 & 4.62 & 5.76 \\
\hline Total $\left(U_{T}\right)$ & 60.8 & 79.16 & 59.75 & 76.36 & 59.75 & 76.36 & 79.31 & 58.77 & 59.75 & 76.36 \\
\hline
\end{tabular}

TABLE 5. Kink energy for $I_{1}, I_{2}, I_{3}$

\begin{tabular}{|c|cc|cc|cc|}
\hline & \multicolumn{2}{|c|}{$I_{1}\left(\times 10^{-21}\right)$} & \multicolumn{2}{|c|}{$I_{2}\left(\times 10^{-21}\right)$} & \multicolumn{2}{c|}{$I_{3}\left(\times 10^{-21}\right)$} \\
\cline { 2 - 7 } & $\mathrm{x} 1$ & $\mathrm{x} 2$ & $\mathrm{x} 1$ & $\mathrm{x} 2$ & $\mathrm{x} 1$ & $\mathrm{x} 2$ \\
\hline \hline$U_{e 1}$ & 8.15 & 7.42 & 6.54 & 6.54 & 8.15 & 7.42 \\
\hline$U_{e 2}$ & 7.42 & 8.15 & 10.08 & 10.08 & 7.42 & 8.15 \\
\hline$U_{e 3}$ & 11.52 & 16.21 & 11.52 & 16.21 & 11.52 & 16.21 \\
\hline$U_{e 4}$ & 7.59 & 11.52 & 7.59 & 11.52 & 7.59 & 11.52 \\
\hline$U_{e 5}$ & 8.15 & 14.81 & 8.15 & 14.81 & 8.15 & 14.81 \\
\hline$U_{e 6}$ & 5.62 & 8.15 & 5.62 & 8.15 & 5.62 & 8.15 \\
\hline$U_{e 7}$ & 5.76 & 7.14 & 5.76 & 7.14 & 5.76 & 7.14 \\
\hline$U_{e 8}$ & 4.62 & 5.76 & 4.62 & 5.76 & 4.62 & 5.76 \\
\hline$U_{e 9}$ & 9.04 & 7.25 & 9.04 & 7.25 & 9.04 & 7.25 \\
\hline$U_{e 10}$ & 7.25 & 6.22 & 7.25 & 6.22 & 7.25 & 6.22 \\
\hline$U_{e 11}$ & 9.33 & 9.33 & 9.33 & 9.33 & 9.33 & 9.33 \\
\hline$U_{e 12}$ & 9.33 & 9.33 & 9.33 & 9.33 & 9.33 & 9.33 \\
\hline$U_{e 13}$ & 6.22 & 7.25 & 6.22 & 7.25 & 6.22 & 7.25 \\
\hline$U_{e 14}$ & 7.25 & 9.04 & 7.25 & 9.04 & 7.25 & 9.04 \\
\hline$U_{e 15}$ & 4.26 & 4.9 & 4.26 & 4.9 & 4.26 & 4.9 \\
\hline$U_{e 16}$ & 4.9 & 5.97 & 4.9 & 5.97 & 4.9 & 5.97 \\
\hline Total $\left(U_{T}\right)$ & 116.41 & 138.45 & 117.46 & 139.5 & 116.41 & 138.45 \\
\hline & & & & & & \\
\hline
\end{tabular}


Simulation Results
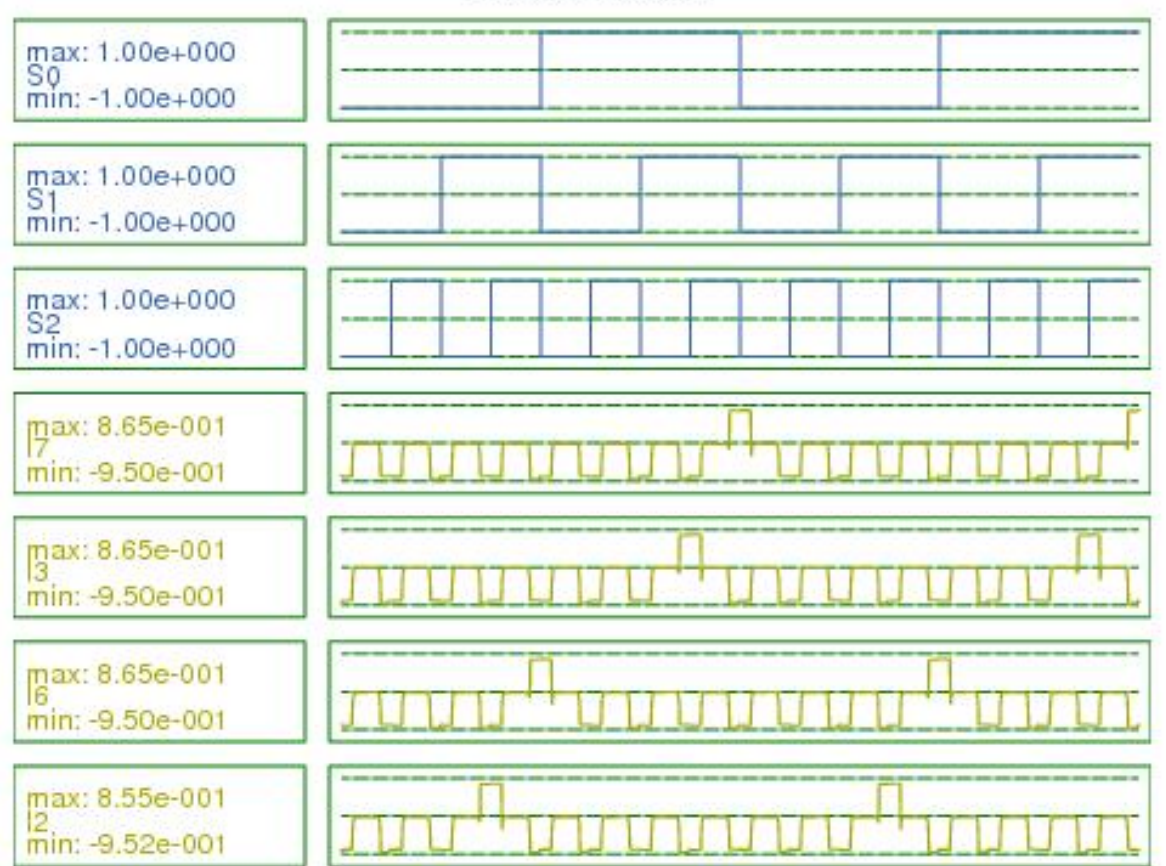

min: $-9.52 \mathrm{e}-001$

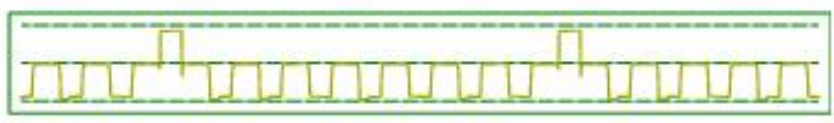

$\max : 8.58 \mathrm{e}-001$

min: $-9.51 \mathrm{e}-001$
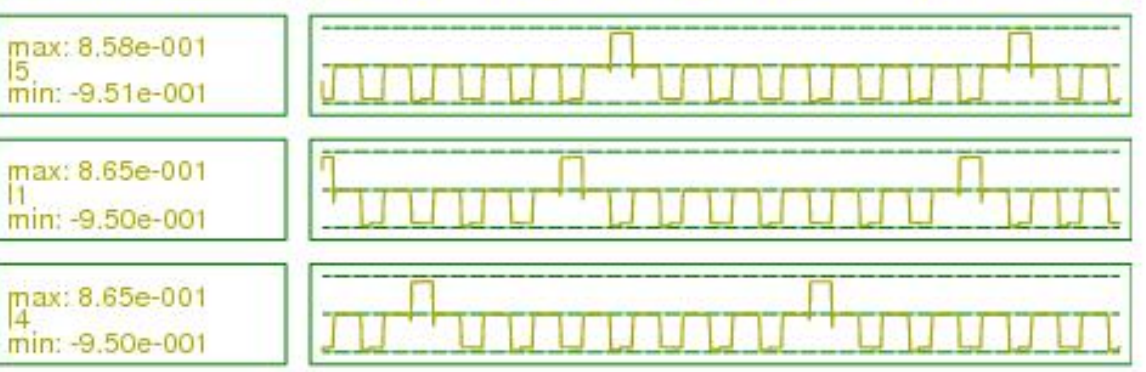

max: $8.58 \mathrm{e}-001$

min: $-9.51 e-001$

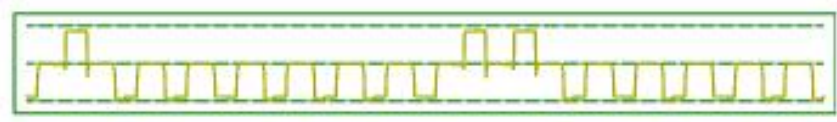

max: $9.80 \mathrm{e}-022$

min: 3.80 e- 023

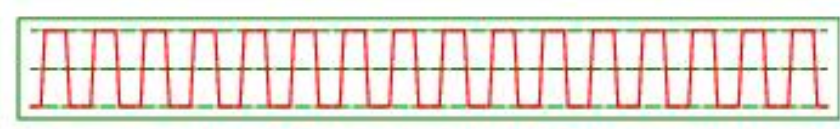

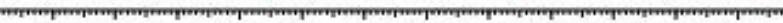
$\begin{array}{lllllllllllll}0 & 1000 & 2000 & 3000 & 4000 & 5000 & 6000 & 7000 & 8000 & 9000 & 10000 & 11000 & 12000\end{array}$

FIG. 11. Output of proposed 3:8 decoder circuit

TABLE 6. Observations for 2:4 decoders

\begin{tabular}{|c|c|c|c|}
\hline Decoder circuit & No of cells & No of layers & Area $\left(\mu \mathrm{m}^{2}\right)$ \\
\hline \hline$[15]$ & 62 & 3 & 0.03 \\
\hline$[16]$ & 88 & 3 & 0.06 \\
\hline$[17]$ & 212 & 1 & 0.25 \\
\hline$[18]$ & 93 & 1 & 0.09 \\
\hline proposed & 50 & 1 & 0.01 \\
\hline
\end{tabular}



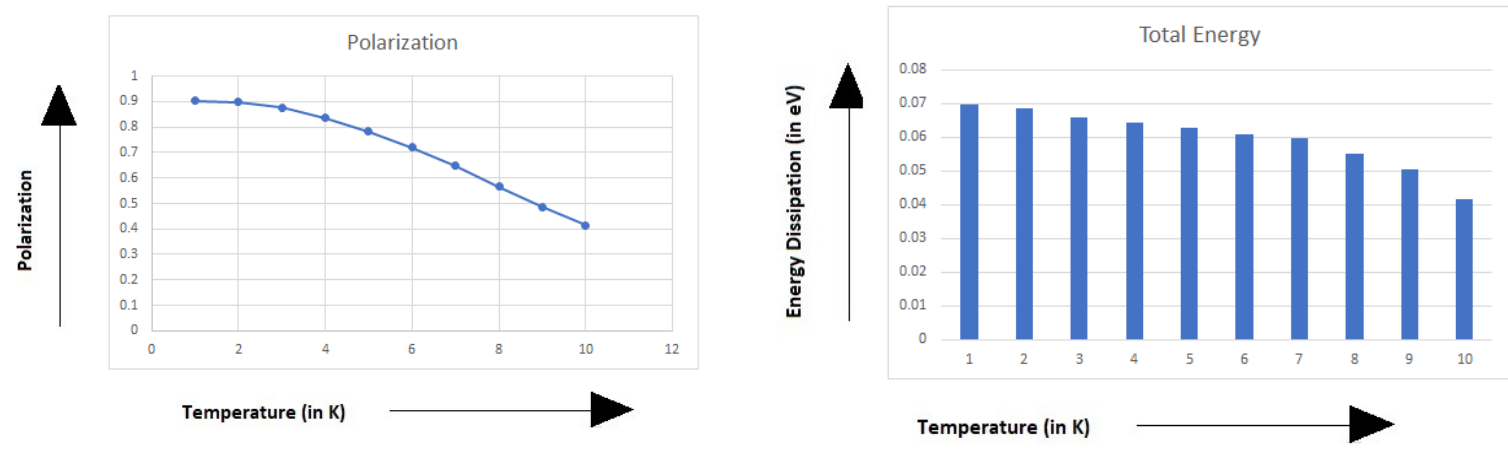

FIG. 12. Polarization (a) or energy dissipation (b) vs temperature

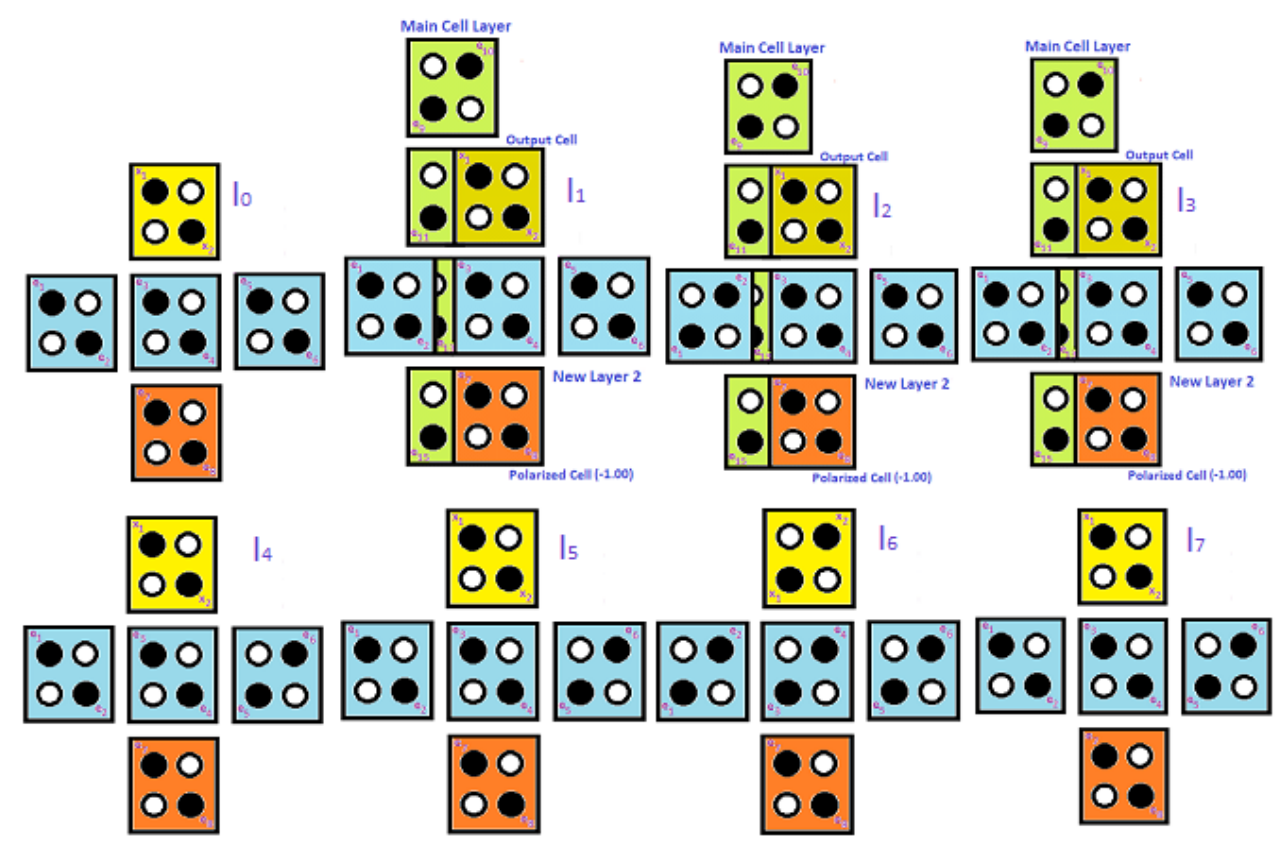

FIG. 13. Reference Diagram for kink energy calculation $\left(I_{0}, I_{1}, I_{2}, I_{3}\right.$ (top row left to right); $I_{4}$, $I_{5}, I_{6}, I_{7}$ (bottom row left to right))

TABLE 7. Observations for 3:8 decoders

\begin{tabular}{|c|c|c|c|}
\hline Decoder circuit & No of cells & No of layers & Area $\left(\mu \mathrm{m}^{2}\right)$ \\
\hline \hline$[15]$ & 136 & 3 & 0.04 \\
\hline$[18]$ & 312 & 1 & 0.1 \\
\hline proposed & 168 & 3 & 0.04 \\
\hline
\end{tabular}




\section{References}

[1] Zoka S., Gholami M.A. Novel efficient full adder-subtractor in QCA nanotechnology. Int. Nano Lett., 2019,9 , P. 51-54.

[2] Sen B., Rajoria A., Sikdar B.K. Design of Efficient Full Adder in Quantum-Dot Cellular Automata. The Scientific World Journal, 2013, 250802.

[3] Jaiswal R., Sasamal T.N. Efficient design of full adder and subtractor using 5-input majority gate in QCA. Tenth International Conference on Contemporary Computing (IC3), 2017, P. 1-6.

[4] Aishwarya Tambe, Snehal Bhakre, Sankit Kassa. Design and Analysis of $(2 \times 1)$ and $(4 \times 1)$ Multiplexer Circuit in Quantum dot Cellular Automata Approach. Int. Journal of Innovative Technology and Exploring Engineering, 2019, 8 (6S3).

[5] Firdous Ahmad. An Optimal Design of QCA Based 2n:1/1:2n Multiplexer/Demultiplexer and Its Efficient Digital Logic Realization. Microprocessors and Microsystems, 2018, 56, P. 64-75.

[6] Khan A., Mandal S., Nag S., Chakrabarty R. Efficient multiplexer design and analysis using quantum dot cellular automata. IEEE Distributed Computing, VLSI, Electrical Circuits and Robotics (DISCOVER), 2016, P. 163-168.

[7] Debarka Mukhopadhyay, Paramartha Dutta. Quantum Cellular Automata based Novel Unit 2:1 Multiplexer. Int. Journal of Computer Applications, 2012, 43 (2), P. 22-25.

[8] Kianpour M., Sabbaghi-Nadooshan R. A Novel Modular Decoder Implementation in Quantum-Dot Cellular Automata (QCA). Int. Conference on Nanoscience, Technology and Societal Implications, Bhubaneswar, 2011, P. 1-5.

[9] Mingliang Zhang, Wenqiang Li, et al. A Programmable Hamming Encoder/Decoder System Design with Quantum-dot Cellular Automata. 3rd Int. Conference on Electronic Information Technology and Computer Engineering, 2019, P. 1338-1345.

[10] Angizi S., Sarmadi S., Sayedsalehi S., Navi K. Design and evaluation of new majority gate-based RAM cell in quantum-dot cellular automata. Microelectronics Journal, 2015, 46 (1), P. 43-51.

[11] Milad Bagherian Khosroshahy, Mohammad Hossein Moaiyeri, Keivan Navi, Nader Bagherzadeh. An energy and cost efficient majority-based RAM cell in quantum-dot cellular automata. Results in Physics, 2017, 7, P. 3543-3551.

[12] Radhouane Laajimi. Nanoarchitecture of Quantum-Dot Cellular Automata (QCA) Using Small Area for Digital Circuits. In Advanced Electronic Circuit - Principles, Architectures and Applications on Emerging Technologies, IntechOpen, London, 2018.

[13] Naqvi S.R., Akram T., et al. A dynamically reconfigurable logic cell: from artificial neural networks to quantum-dot cellular automata. Appl. Nanosci., 2018, 8, P. 89-103.

[14] Lent C.S., Tougaw P.D. A device architecture for computing with quantum dots. Proceedings of the IEEE, 1997, 85 (4), P. $541-557$.

[15] Abbasizadeh A., Mosleh M. Ultra dense 2-to-4 decoder in quantum-dot cellular automata technology based on MV32 gate. ETRI Journal, 2020, 42 (6), P. 912-921.

[16] Seyedi S., Navimipour N.J. An Optimized Three-Level Design of Decoder Based on Nanoscale Quantum-Dot Cellular Automata. Int. Journal of Theoretical Physics, 2018, 57, P. 2022-2033.

[17] Kumar M., Sasamal T.N. An Optimal design of 2-to-4 Decoder circuit in coplanar Quantum-dot cellular automata. Energy Procedia, 2017, 117, P. 450-457.

[18] De D., Purkayastha T., Chattopadhyay T. Design of QCA based Programmable Logic Array using decoder. Microelectronics Journal, 2016, 55, P. 92-107.

[19] QCADesigner 2.0. URL: https://qcadesigner.software.informer.com/2.0/. 